\title{
Procedimientos endoscópicos y terapias antitrombóticas. Una visión actual
}

\section{The Current View of Endoscopic procedures and Antithrombotic Therapies}

\author{
Robin Germán Prieto Ortiz, MD, ${ }^{1}$ Bernardo Borráez Segura, MD, ${ }^{2}$ Martha L. Suárez Acuña, MD, ${ }^{3}$ Diego M. Aponte Martín, MD, ${ }^{4}$ \\ Ricardo Oliveros Wilches, MD. ${ }^{5}$
}

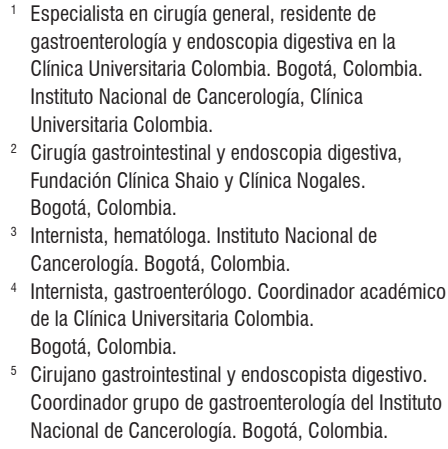
gastroenterología y endoscopia digestiva en la Clínica Universitaria Colombia. Bogotá, Colombia Instituto Nacional de Cancerología, Clínica Universitaria Colombia.

2 Cirugía gastrointestinal y endoscopia digestiva, Fundación Clínica Shaio y Clínica Nogales. Bogotá, Colombia.

3 Internista, hematóloga. Instituto Nacional de Cancerología. Bogotá, Colombia.

${ }^{4}$ Internista, gastroenterólogo. Coordinador académico de la Clínica Universitaria Colombia. Bogotá, Colombia.

5 Cirujano gastrointestinal y endoscopista digestivo. Coordinador grupo de gastroenterología del Instituto Nacional de Cancerología. Bogotá, Colombia.

\section{Resumen}

En los últimos años se ha generado un avance importante en el tratamiento de las patologías procoagulantes con antiguos y nuevos medicamentos y se ha logrado un importante avance en los métodos diagnósticos y terapéuticos en la endoscopia digestiva. Además, la expectativa de vida de la población mundial se ha incrementado de forma considerable, por lo que más frecuentemente el gastroenterólogo debe realizar procedimientos endoscópicos en pacientes de mayor edad, quienes presentan comorbilidades por las que reciben terapias antitrombóticas, especialmente por patologías cardiovasculares.

En la literatura aparecen muchas guías de manejo avaladas por importantes asociaciones, en las que se orienta sobre la realización de procedimientos endoscópicos en los pacientes sometidos a terapias antitrombóticas, procedimientos que representan un determinado riesgo de sangrado o trombosis en caso de la suspensión de los medicamentos, pero no se han realizado estudios prospectivos o controlados que permitan formular protocolos para las diversas técnicas endoscópicas diagnósticas o terapéuticas en estos pacientes. El gastroenterólogo debe realizar un balance entre el riesgo hemorrágico y embólico para determinar cuál es el momento más indicado para realizar un procedimiento endoscópico en estos pacientes; por lo tanto, es de gran importancia que tenga conocimiento de este tema.

Se presenta una revisión actualizada de la literatura y las más recientes recomendaciones de la sociedad europea de gastroenterología.

\section{Palabras clave}

Terapia antitrombótica, endoscopia digestiva, antiagregantes, anticoagulantes.

\section{Abstract}

In recent years, treatment of pathologies related to hypercoagulation has advanced significantly as the result of the use of old and new drugs. In addition, significant advances have been achieved in diagnostic and therapeutic methods in digestive endoscopy. On the other hand, the life expectancy of the world population has increased considerably, so gastroenterologists must perform endoscopic procedures more frequently in older patients who present comorbidities for which they receive antithrombotic therapies, especially for cardiovascular diseases.

Many treatment guides produced by important medical associations are available. They are oriented to the performance of endoscopic procedures in patients undergoing antithrombotic therapies. These patients are at risk of bleeding during or after these procedures, and they are at risk of thrombosis when medication is suspended. Nevertheless, no prospective or controlled studies have been performed to guide formulation of protocols for the various diagnostic and therapeutic endoscopic techniques that might be used for these patients. Gastroenterologists must balance the risks of bleeding and embolism to determine the most appropriate time to perform an endoscopic procedure in these patients. Therefore, it is of great importance that you have knowledge of this topic.

We present an updated review of the literature and the most recent recommendations of the European society of gastroenterology.

\section{Keywords}

Antithrombotic therapy, digestive endoscopy, antiplatelet drug (antiaggregant), anticoagulants. 


\section{INTRODUCCIÓN}

Como resultado de la mejora en los sistemas de salud, generado en parte por el avance en los métodos diagnósticos y terapéuticos, la expectativa de vida en la población mundial se ha incrementado de forma considerable. Adicionalmente, cada vez más frecuentemente, el gastroenterólogo debe realizar procedimientos endoscópicos en pacientes de mayor edad, quienes presentan comorbilidades por las que reciben terapias antitrombóticas, especialmente por patologías cardiovasculares. A comienzos de esta década y de acuerdo con un estudio publicado en la literatura norteamericana, se consideraba que cerca del $70 \%$ de los pacientes con enfermedad cardiovascular recibían ácido acetilsalicílico (ASA), hasta un 18\% requería el uso de doble terapia antiagregante y un $6 \%$ tenía indicación de terapia combinada (ASA, anticoagulante oral) (1).

En este grupo de pacientes el riesgo de sangrado digestivo se asocia con factores como el consumo de antiagregantes o anticoagulantes, la edad avanzada, el género femenino, el antecedente de úlcera péptica, el antecedente de choque cardiogénico y la no formulación de un inhibidor de bomba de protones (IBP) cuando está indicado. Según algunos estudios, se considera que la mortalidad hospitalaria debida a eventos cardiovasculares trombóticos es cercana al $4 \%$ y la relacionada con sangrado del tracto gastrointestinal (TGI) es del $0,15 \%(2,3)$.

¿Cuál es el momento más indicado para realizar un procedimiento endoscópico en los pacientes sometidos a terapia antiembólica? El gastroenterólogo debe realizar un balance entre el riesgo hemorrágico y el riesgo embólico y frecuentemente debe contar con el apoyo del hematólogo, cardiólogo y cirujano cardiovascular para determinar el mejor momento para la realización del procedimiento.

A pesar de la importancia de este tema, no se han realizado estudios prospectivos o controlados que permitan formular protocolos para la realización de las diversas técnicas endoscópicas diagnósticas o terapéuticas en pacientes con terapia antiembólica, pero sí existen muchas guías de manejo formuladas por las más importantes sociedades científicas que orientan en la atención de este tipo de pacientes $(4,5)$.

\section{NUEVA CASCADA DE LA COAGULACIÓN}

Ante el incremento en la prescripción de los medicamentos antiagregantes y anticoagulantes es de gran importancia que el gastroenterólogo tenga presente la fisiología de la coagulación y la farmacodinamia de los medicamentos empleados.

En 1964, MacFarlane describió la cascada de la coagulación en la que, a través de 2 vías (la intrínseca [factores XII,
XI, IX, VIII y V] y la extrínseca [factor tisular y factor VII], que ulteriormente se unen en una vía común [factor $\mathrm{X}]$ ), los diversos factores de la coagulación interactúan para que finalmente y a través de la protrombinasa ocurra la conversión de la protrombina a trombina y, mediante esta última, del fibrinógeno a la fibrina. En la cascada clásica, las plaquetas no tienen tanta importancia y desarrollan su función de forma independiente (6).

A partir de 1994 y de forma casi simultánea, se planteó un nuevo proceso de la coagulación publicado en 2 estudios, en los que se modifica la cascada clásica y se postulan enunciados importantes como el de que las 2 vías de la coagulación van unidas casi desde el inicio del proceso, debido a que el complejo factor tisular-factor VIII participa también en la activación del factor IX, y que el proceso ocurre en 3 fases consecutivas casi simultáneas, más que en forma de cascada. La primera fase es la fase inicial (se producen pequeñas cantidades de trombina a partir de la activación del factor X de forma indirecta -activación del factor IX-y de forma directa, ambos favorecidos por el complejo factor tisular-factor VII). La segunda es la fase de amplificación (las plaquetas liberan fosfolípidos ácidos que, junto con el calcio de la sangre, activan los factores XI, IX, VIII y V; que por mecanismos quimiotácticos son atraídos a la superficie de las plaquetas, donde ocurren procesos de retroalimentación y multiplicación). Y la tercera es la fase de propagación (se activan grandes cantidades de factor $\mathrm{X}$, se forma el complejo protrombinasa, que convierte la protrombina en trombina y, por esta última, el fibrinógeno en fibrina. Todo este proceso ocurre en la superficie de la plaqueta y de forma exponencial). En este nuevo proceso planteado se da especial importancia a la función de las plaquetas y de la trombina, que actúan especialmente en las 2 fases finales. Este nuevo proceso de la coagulación ha sido aceptado desde 2007 por la sociedad europea de cardiología (7-9).

A lo largo de los años se han realizado muchas investigaciones con el fin de impedir el proceso de la coagulación cuando está indicado. La inhibición de la protrombina mediante fármacos contra la vitamina $\mathrm{K}$ (warfarina, acenocumarol) es la terapia más utilizada en la prevención crónica de procesos trombóticos. La inhibición del factor VIII, mediante las diferentes formas de heparina, sigue siendo utilizada con éxito por su fácil control y escaso riesgo hemorrágico. Los nuevos anticoagulantes orales (NACO) actúan sobre el factor $\mathrm{X}$ y directamente sobre la trombina. Actualmente, también es claro que la combinación de fármacos inhibidores de la trombina y de la actividad plaquetaria en dosis correspondientes al nivel inferior del rango terapéutico puede conseguir un efecto antitrombótico efectivo sin riesgo de hemorragias (6). 


\section{MEDICAMENTOS ANTIEMBÓLICOS EMPLEADOS}

- Antiagregantes plaquetarios

- Inhibidores enzimáticos

- Inhibidores de la ciclooxigenasa (ASA)

- Inhibidores de la fosfodiesterasa (dipiridamol)

- Inhibidores de receptores específicos

- Inhibidores de receptores de difosfato de adenosina (ADP) (clopidogrel, ticagrelor, prasugrel)

- Antagonistas IIb/IIIa (tirofibán, abciximab, trigramin)

- Anticoagulantes

- Heparinas

- Heparina no fraccionada (HNF)

- Heparina de bajo peso molecular (HBPM) (enoxaparina, dalteparina, fondaparinux)

- Anticoagulantes orales

- Inhibidores de la vitamina K (warfarina)

- Inhibidores selectivos delfactorXa (rivaroxabán, apixabán)

- Inhibidores directos de la trombina (dabigatrán)

\section{Antiagregantes plaquetarios}

\section{Ácido acetilsalicilico (ASA)}

Es uno de los medicamentos más conocidos en cuanto a su farmacodinamia y uno de los más usados a lo largo de la historia, con amplia indicación en la patología cardiovascular y que actúa inhibiendo la ciclooxigenasa 1, por lo que bloquea de forma selectiva la síntesis a nivel plaquetario del tromboxano A2 y bloquea de forma irreversible la función plaquetaria. Su efecto se contrarresta mediante la administración de plaquetas (10).

\section{Tienopiridinas}

Son antagonistas del receptor ADP que impiden de forma selectiva e irreversible la agregación y activación plaquetarias. No tienen antídoto y ante cualquier tipo de procedimiento endoscópico, independiente del riesgo tromboembólico, se recomienda su suspensión. Cuando el paciente tiene un alto riesgo tromboembólico se recomienda remplazarlo por ASA a dosis de $100 \mathrm{mg}$ día, hasta que se pueda reiniciar la tienopiridina.

Cuando el paciente recibe doble terapia, se debe suspender la tienopiridina y continuar con la administración de ASA. Las tienopiridinas no tienen antídoto, por lo que, ante la necesidad de revertir su efecto, se debe plantear la transfusión plaquetaria según el riesgo hemorrágico del procedimiento endoscópico a realizar (11).

\section{Clopidogrel}

Se administra a dosis de $75 \mathrm{mg}$ día, logra una inhibición del 25\%-30\% al segundo día y una máxima inhibición entre el quinto y séptimo día. Estos tiempos se acortan si se administran dosis superiores. Cada día que se interrumpe la administración de clopidogrel se consigue una regeneración plaquetaria del 10\%-14\%. Debe ser suspendido 5 días antes del procedimiento endoscópico.

\section{Ticagrelor}

Tiene un efecto inhibitorio más rápido que el clopidogrel; se administra a dosis de $90 \mathrm{mg} / 12$ horas y logra un pico máximo a las 2-4 horas. Su acción desciende rápidamente luego de las 72 horas y hasta los 5 días. Debe suspenderse 5 días antes del procedimiento endoscópico.

\section{Prasugrel}

Es 10-100 veces más potente que el clopidogrel; se administra en dosis única diaria de $10 \mathrm{mg}$ y se debe suspender 7-10 días antes del procedimiento endoscópico.

Se debe recordar que los procedimientos endoscópicos de alto riesgo requieren cifras plaquetarias por encima de 50000 unidades, mientras que los de bajo riesgo precisan de valores de plaquetas superiores a 20000 . Las plaquetas se deben administrar antes y durante el procedimiento $y$ la dosis recomendada es de una unidad por cada $10 \mathrm{~kg}$ de peso. Por cada unidad de plaquetas que se administre, se logra un incremento de 5000 a 10000 plaquetas, lo que se denomina rendimiento plaquetario (12).

\section{Anticoagulantes orales}

Inhiben los factores dependientes de vitamina $\mathrm{K}$ y requieren la antitrombina para actuar; tienen un reducido margen terapéutico, por lo que requieren una permanente monitorización mediante la determinación del índice internacional normalizado (INR); ante procedimientos de bajo riesgo hemorrágico se recomienda continuar su uso, pero se debe suspender ante procedimientos de alto riesgo. Cuando se realizan procedimientos de bajo riesgo trombótico se debe suspender 3-5 días antes del mismo verificando valores de INR $<1,5$; cuando existe alto riesgo trombótico se debe realizar terapia puente con las $\operatorname{HBPM}(13,14)$.

En casos de urgencias se debe buscar la reversión de la anticoagulación (INR 1,5-2,5) mediante la administración de vitamina $\mathrm{K}$ (fitomenadiona $10 \mathrm{mg}$ ), concentrado de complejo protrombínico como segunda opción, factor VII recombinante y plasma fresco si es la única terapéutica disponible, a dosis de 10 a $30 \mathrm{~mL} / \mathrm{kg}$ (12). 


\section{Nuevos anticoagulantes orales (NACO)}

Algunos estudios han demostrado un mejor efecto anticoagulante con los NACO en la prevención de accidentes cerebrovasculares y de eventos embólico-sistémicos en comparación con la warfarina. Tienen una acción directa sin necesitar de la antitrombina, no requieren monitorización por su acción más estable y su dosis no requiere ajustes. El efecto anticoagulante se logra y cesa más rápido luego de su administración o suspensión, en comparación con el de la warfarina. No existen antídotos que reviertan su acción. Algunos estudios mencionan un mayor riesgo de sangrado con el uso de los NACO, especialmente con el dabigatrán $(11,15)$.

La Sociedad Europea de Anestesiología establece que no es necesario suspender los NACO para endoscopias altas ni para colonoscopias, incluso si está programada la realización de biopsias (16).

Cuando se realiza un procedimiento en pacientes que reciben fármacos con administración de 2 dosis diarias (dabigatrán y apixabán), la endoscopia debe realizarse más de 10 horas luego de la última toma; mientras que cuando reciben rivaroxabán, que se administra una vez al día, se recomienda su suspensión por lo menos un día antes del procedimiento. En procedimientos de alto riesgo se recomienda evaluar la depuración de creatinina: si es $>50 \mathrm{~mL} /$ min, suspender 1-2 días antes; si es menor, suspender 3-5 días antes. Se recomienda reiniciar el medicamento al día siguiente del procedimiento (17).

\section{Dabigatrán}

Actúa directamente sobre la trombina (Factor IIa), tiene una vida media de 9-17 horas que varía según la edad y la función renal, se administra en dosis de $75 \mathrm{mg}$ cada 12 horas, se absorbe en el intestino proximal y se elimina por vía renal.

\section{Rivaroxabán}

Impide la formación de trombina al inhibir de forma directa el factor Xa, la vida media es 5-9 horas en pacientes jóvenes y de 11-13 horas en pacientes mayores, se administra en dosis de $10 \mathrm{mg} /$ día. Su absorción a nivel gastrointestinal no se ve alterada por el consumo de alimentos, tiene metabolismo hepático y excreción renal, por lo que su uso está contraindicado en pacientes con cirrosis avanzada e insuficiencia renal severa.

\section{Apixabán}

Inhibe de forma irreversible el factor $\mathrm{Xa}$, tiene una vida media de 8 a 15 horas, se administra cada 12 horas a dosis de $2,5 \mathrm{mg}$. Se absorbe a nivel gastrointestinal y se metaboliza a nivel hepático, una tercera parte del fármaco se elimina por las heces sin ser absorbido y una cuarta parte se elimina por excreción renal. No se recomienda en pacientes renales o con hepatopatía (11).

\section{Terapia puente}

Está indicada en portadores de válvulas protésicas (reemplazo valvular mitral, 2 o más válvulas mecánicas, reemplazo de válvula aórtica bivalva o reemplazo valvular aórtico con otros factores de riesgo), fibrilación auricular no valvular (con antecedente de evento embólico, trombo intracardíaco, puntaje de CHADS2 score $\geq 4$ ) y tromboembolismo venoso (en 3 meses previos o trombofilia severa [deficiencia de proteína $\mathrm{C}$, proteína $\mathrm{S}$ o antitrombina; síndrome antifosfolipídico; homocigoto para factor $\mathrm{v}$ de Leiden; homocigoto para la mutación del gen de protrombina G20210A; o componente heterocigoto para los 2 genes]) (18).

\section{CONSIDERACIONES EN CASO DE SANGRADO}

La realización de una endoscopia de vías digestivas (EVD) debe ser considerada de forma urgente en pacientes que presentan sangrados digestivos con disminución de la hemoglobina $(\mathrm{Hb})$ en más de $2 \mathrm{~g}$ y en los pacientes con signos evidentes de sangrado, como hematemesis, hematoquecia o melenas. Cuando estos pacientes reciben terapia antitrombótica, presentan un incremento $>1,5 \%$ en el riesgo de sangrado luego de la realización de la endoscopia, más aún si hay antecedentes de insuficiencia renal, hepatopatía o cáncer.

Cuando los pacientes reciben ASA y presentan un sangrado que requiera la realización de una EVD, se debe primero compensar hemodinámicamente al paciente mediante el uso de líquidos endovenosos (LEV), inotrópicos y transfusión de plaquetas cuando sea necesario. No se ha observado un incremento del riesgo de sangrado en pacientes sometidos a EVD y en cambio sí se ha observado un incremento de la mortalidad a 30 días en pacientes de alto riesgo cardiovascular en quienes se ha suspendido el ASA.

En pacientes que reciben warfarina puede ser necesaria la administración de vitamina $\mathrm{K}$ y plasma fresco congelado, se considera que la EVD se puede realizar con valores de INR $<2,5$, aunque por seguridad se prefiere tener cifras $<1,5$.

En pacientes que reciben NACO y presentan sangrado con requerimiento de EVD, y en tanto sea posible por las condiciones del paciente, se deberá administrar abundantes LEV ya que la eliminación de estos medicamentos es por vía renal. Se debe tener en cuenta la administración de factor VIIa o de concentrados de complejo de protrombina activado, especialmente en caso de consumo de dabigatrán, o no activado en caso de consumo de rivaroxabán o apixabán $(18,19)$.

Una vez controlado el sangrado se puede reiniciar la administración del tratamiento antitrombótico, de ser posible 
el mismo día del procedimiento. La recomendación en los pacientes que reciben doble antiagregación es que nunca se suspendan el ASA, las tienopiridinas se deben suspender entre 5-7 días antes del procedimiento y se administran nuevamente una vez se haya controlado el sangrado. las HBPM se pueden reiniciar en las primeras 24 horas si se trata de un procedimiento de bajo riesgo de sangrado o 72 horas después en caso de procedimientos de alto riesgo de sangrado (20).

\section{Riesgo tromboembólico frente al riesgo de sangrado}

En el momento de realizar un procedimiento endoscópico en pacientes con terapia antiembólica, es necesario realizar un balance entre el riesgo de suspender la medicación (que podría causar un tromboembolismo en el paciente) y el riesgo de no suspenderlo (que podría incrementar el riesgo de sangrado). De acuerdo con la literatura, las principales patologías de alto riesgo de tromboembolismo son (11):

- Fibrilación auricular no valvular: la anticoagulación en estos pacientes está indicada con la existencia de uno solo de los siguientes criterios: insuficiencia cardíaca congestiva reciente (1), hipertensión arterial (1), edad $\geq 75$ años (2), diabetes mellitus (1), accidente cerebrovascular (ACV) o accidente isquémico transitorio (2), enfermedad vascular previa (1), edad 65-75 años (1) y género femenino (1). Estos criterios están agrupados en la escala de CHADS2 O CHA2DS2-VASc que determinan el riesgo de ACV en pacientes sin terapia antitrombótica. Cada criterio tiene un valor (entre paréntesis) y a mayor puntaje, mayor porcentaje de riesgo de tromboembolismo $(21,22)$.

- Valvulopatías con prótesis metálicas: cuando se trata de una prótesis metálica aórtica bivalva sin otros factores asociados, existe un riesgo que se considera bajo (riesgo anual $<5 \%)$ para que ocurran eventos tromboembólicos. Si además el paciente presenta fibrilación auricular, el riesgo se eleva a moderado (5\%-10\%) y el riesgo se considera alto (>10\%) en caso de cualquier prótesis metálica mitral, tricúspide, aórtica diferente a bivalva, múltiples prótesis o el antecedente de un evento cardioembólico (23).

- Enfermedad cardiovascular con inserción de stent coronarios: los pacientes con antecedente de colocación de stent convencionales tienen un mayor riesgo de trombosis si se suspende la doble antiagregación durante las primeras 6 semanas; si se trata de stents medicados, el mayor riesgo de trombosis existe durante los primeros 3 a 6 meses; sin embargo, se considera que existe alto riesgo de trombosis, si la suspensión de la medicación se realiza durante el primer año luego de la colocación del stent (24).

- Trombosis venosa profunda: durante los primeros 3 meses luego de ocurrido el evento, existe un riesgo alto $(>10 \%)$ de que ocurran nuevos eventos tromboembó- licos. Entre los 3 y los 12 meses el riesgo está entre el 5\% y el 10\%, y cuando ha pasado más de un año el riesgo de eventos baja al 5\% (25).

\section{Riesgo de sangrado}

Diversas asociaciones y sociedades han clasificado los procedimientos endoscópicos diagnósticos o terapéuticos de acuerdo con el riesgo de presentar sangrado durante la realización del mismo en pacientes que reciben la terapia antiembólica y, de acuerdo con estos, han formulado sus recomendaciones en cuanto al manejo de la anticoagulación.

En las Tablas 1 y $\mathbf{2}$ se muestran los procedimientos endoscópicos y su riesgo de sangrado en pacientes que reciben terapia antiembólica de acuerdo con las clasificaciones de la Sociedad Española de Patología Digestiva (SEPD) y la Sociedad Europea de Endoscopia Gastrointestinal (ESGE).

Tabla 1. Riesgo de sangrado según el procedimiento SEPD, Sociedad Española de Endoscopia Digestiva (SEED), Sociedad Española de Trombosis y Hemostasia (SETH) y Sociedad Española de Cardiología (SEC), 2015

\begin{tabular}{ll}
\hline \multicolumn{1}{c}{ Bajo riesgo hemorrágico } & \multicolumn{1}{c}{ Alto riesgo hemorrágico } \\
\hline $\begin{array}{l}\text { Endoscopia diagnóstica con o sin } \\
\text { biopsia }\end{array}$ & Polipectomía \\
$\begin{array}{l}\text { CPRE diagnóstica } \\
\text { Inserción de prótesis biliares sin } \\
\text { esfinterotomía } \\
\text { Ecoendoscopia }\end{array}$ & $\begin{array}{l}\text { Coagulación y ablación con láser } \\
\text { Esinterotomía }\end{array}$ \\
& Dilatación de estenosis benignas \\
onteroscopia de pulsión & Colocación de PEG \\
& $\begin{array}{l}\text { Ecoendoscopia con punción de } \\
\text { lesión quística }\end{array}$ \\
& Enteroscopia con balón \\
& Resección mucosa, disección \\
& submucosa, ampulectomía \\
\hline
\end{tabular}

CPRE: colangiopancreatografía retrógrada endoscópica; PEG: polietilenglicol. Tomado de: Alberca de las Parras F et al. Rev Esp Enferm Dig. 2015;107(5):289-306.

De acuerdo con algunos estudios, se considera que un procedimiento es de bajo riesgo si la probabilidad de sangrado es $<1,5 \%$ (18), mientras que para otros grupos el riesgo de sangrado debe ser $<1,0 \%$ para que se considere un procedimiento de bajo riesgo de sangrado (11).

En general, las diversas guías recomiendan que cuando un paciente tiene una patología de alto riesgo tromboembólico y requiere un procedimiento considerado de bajo riesgo de sangrado, se pueden continuar los medicamentos, incluso si se requiere la toma de biopsias. La incidencia de sangrado no varía mucho en comparación con la de los pacientes a los que se les suspende la terapia antitrombótica (27). 
Tabla 2. Riesgo de sangrado de los procedimientos según las guías de la British Society of gastroenterology (BSG) y la ESGE 2016 (26)

\begin{tabular}{|c|c|}
\hline Bajo riesgo hemorrágico & Alto riesgo hemorrágico \\
\hline $\begin{array}{l}\text { Endoscopia diagnóstica } \\
\text { con o sin biopsia }\end{array}$ & Polipectomía endoscópica \\
\hline $\begin{array}{l}\text { Colocación de stent biliar } \\
\text { o pancreático }\end{array}$ & CPRE con esfinterotomía \\
\hline \multirow[t]{8}{*}{$\begin{array}{l}\text { Enteroscopia sin } \\
\text { polipectomía }\end{array}$} & $\begin{array}{l}\text { Esfinterotomía más dilatación biliar con } \\
\text { balón }\end{array}$ \\
\hline & Ampulectomía \\
\hline & Resección mucosa, disección submucosa \\
\hline & Dilatación de estenosis \\
\hline & Tratamiento de las várices esofágicas \\
\hline & Colocación de stent \\
\hline & GEP \\
\hline & Ecoendoscopia con punción \\
\hline
\end{tabular}

GEP: Gastrostomía endoscópica percutánea. Tomado de: Veitch AM et al. Gut. 2016;65(3): 374-89.

La recomendación en los procedimientos con alto riesgo hemorrágico y bajo riesgo tromboembólico es la de suspender el tratamiento tromboembólico. En procedimientos de alto riesgo hemorrágico y tromboembólico, la ASGE y la ESGE recomiendan continuar el tratamiento únicamente con ASA. Los procedimientos electivos en este grupo de pacientes deben ser pospuestos hasta completar el tratamiento (18).

\section{RECOMENDACIONES DE LAS GUÍAS ESGE 2016 PARA LA REALIZACIÓN DE ENDOSCOPIA EN PACIENTES CON TERAPIA ANTIPLAQUETARIA O ANTICOAGULANTE}

A continuación, se presenta un resumen de las principales recomendaciones registradas en las guías de la ESGE 2016 (26).

\section{Uso del ASA}

Se recomienda continuar la administración de ASA en todos los procedimientos endoscópicos, excepto la disección endoscópica submucosa (ESD), mucosectomía endoscópica colónica de más de $2 \mathrm{~cm}$, mucosectomía del TGI superior y ampulectomía. En estos casos, se debe evaluar el riesgo-beneficio de la suspensión del medicamento en cada paciente (evidencia de baja calidad o recomendación débil).

\section{Procedimientos de bajo riesgo de sangrado}

- Continuar con la administración de las tienopiridinas, como terapia única o de doble antiagregación (evidencia de baja calidad, recomendación fuerte).

- Continuar con la warfarina (evidencia de baja calidad, recomendación débil). Se debe verificar que el INR no esté por encima del rango terapéutico durante una semana previa al procedimiento (evidencia de baja calidad, recomendación fuerte).

- En los pacientes que reciben los NACO, se recomienda no administrar la dosis de la mañana del día del procedimiento (evidencia de muy baja calidad, recomendación débil) (Figura 1).

\section{Procedimientos de alto riesgo de sangrado}

- Pacientes con bajo riesgo trombótico: suspender la warfarina 5 días antes del procedimiento (evidencia de alta calidad, recomendación fuerte); siempre se debe verificar que el INR esté por debajo de 1,5 (evidencia de baja calidad, recomendación fuerte). Suspender la tienopiridina 5 días previos al procedimiento (evidencia de moderada calidad, recomendación fuerte).

- Pacientes con terapia dual: se recomienda suspender la tienopiridina y continuar con ASA (evidencia de baja calidad, recomendación débil).

- Pacientes con alto riesgo trombótico: se recomienda continuar el ASA y buscar el apoyo de un cardiólogo para establecer el riesgo-beneficio de la suspensión de las tienopiridinas (evidencia de alta calidad, recomendación fuerte).

- En los pacientes de alto riesgo trombótico que reciben warfarina, esta se debe remplazar temporalmente por una HBPM (evidencia de baja calidad, recomendación fuerte).

- Los pacientes que reciben NACO, deben suspender la medicación por lo menos 48 horas antes del procedimiento (evidencia de muy baja calidad, recomendación fuerte). Cuando reciben dabigatrán y tienen una depuración de creatinina entre $30-50 \mathrm{~mL} / \mathrm{min}$, el medicamento se debe retirar 3 días antes del procedimiento (evidencia de baja calidad, recomendación fuerte).

- Los medicamentos suspendidos deben reiniciarse solo después de 48 horas y dependen del riesgo de trombosis o de sangrado (evidencia de calidad moderada, recomendación fuerte) (Figura 2).

\section{Terapia puente}

Se recomienda solo para pacientes que reciben warfarina, ya que cuando se realiza para pacientes que reciben NACO se ha visto que la terapia puente no disminuye el riesgo embólico y en cambio sí incrementa de manera importante el riesgo de sangrado.

\section{Triple terapia antitrombótica}

Se indica especialmente en los pacientes con stents coronarios que reciben terapia dual y que pueden desarrollar fibrilación auricular con indicación de warfarina o NACO, 


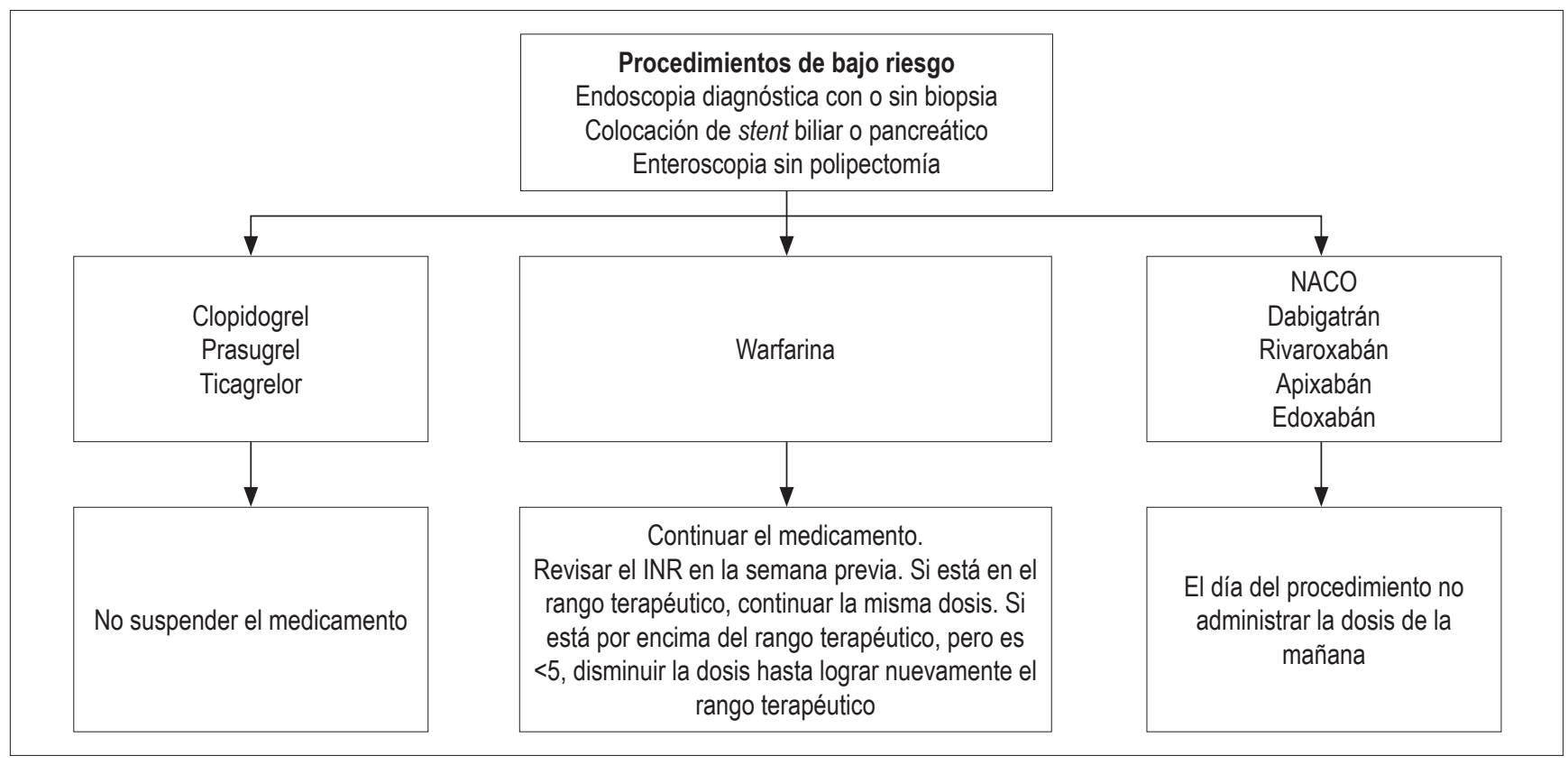

Figura 1. Uso de los medicamentos en procedimientos de bajo riesgo. Modificado de: (26).

o en pacientes con fibrilación auricular crónica que están anticoagulados y que pueden desarrollar un síndrome coronario agudo, que requiere terapia antiplaquetaria dual. Estos pacientes tienen un alto riesgo de sangrado y para la realización de cualquier procedimiento endoscópico deben ser valorados previamente por un cardiólogo u otro especialista según la pertinencia.

\section{Tratamiento del sangrado en pacientes que reciben NACO}

En pacientes que presentan sangrados leves, basta con la suspensión temporal del medicamento. En caso de sangrados graves, puede ser necesaria la hemostasia endoscópica, la administración de glóbulos rojos e incluso la corrección de la coagulopatía (con transfusión de plaquetas y otros productos sanguíneos). Se debe determinar el tiempo de la última ingesta de NACO, así como la vida media a partir de la creatinina sérica y su depuración.

El plasma fresco congelado no revierte el efecto anticoagulante de los NACO de forma considerable y no se han demostrado beneficios clínicos en su uso.

El sulfato de protamina y la vitamina $\mathrm{K}$ no revierten los efectos anticoagulantes de los NACO. No se conoce el efecto de los antifibrinolíticos en sangrado debido a los NACO, pero el uso del ácido tranexámico parece ser una alternativa razonable.
El concentrado de complejo de protrombina y el factor VIIa recombinante (rFVIIa) no han sido evaluados en ensayos clínicos; sin embargo, ante un sangrado que comprometa la vida del paciente se puede usar el concentrado de complejo de protrombina en dosis de 40-50 UI / kg.

\section{Endoscopia diagnóstica y biopsias}

No se ha observado incremento en el riesgo de sangrado en pacientes que son sometidos a biopsias y consumen ASA, clopidogrel o warfarina. Pero tampoco se han realizado estudios serios en pacientes que consumen NACO y que son sometidos a biopsias o en pacientes que requieren múltiples biopsias, como en el seguimiento de esófago de Barrett. Por estas razones y ya que no hay pruebas confiables para determinar el estado de anticoagulación en los pacientes que consumen los NACO, se sugiere no administrar la última dosis del medicamento en la mañana del procedimiento para permitir un margen de seguridad adecuado. Esto se aplica tanto para regímenes de una vez al día como para los de 2 veces al día.

\section{Polipectomía, resección mucosa endoscópica (EMR) y ESD}

El tamaño del pólipo es el factor de riesgo más importante en cuanto a pólipos colónicos se refiere. También se ha impli- 


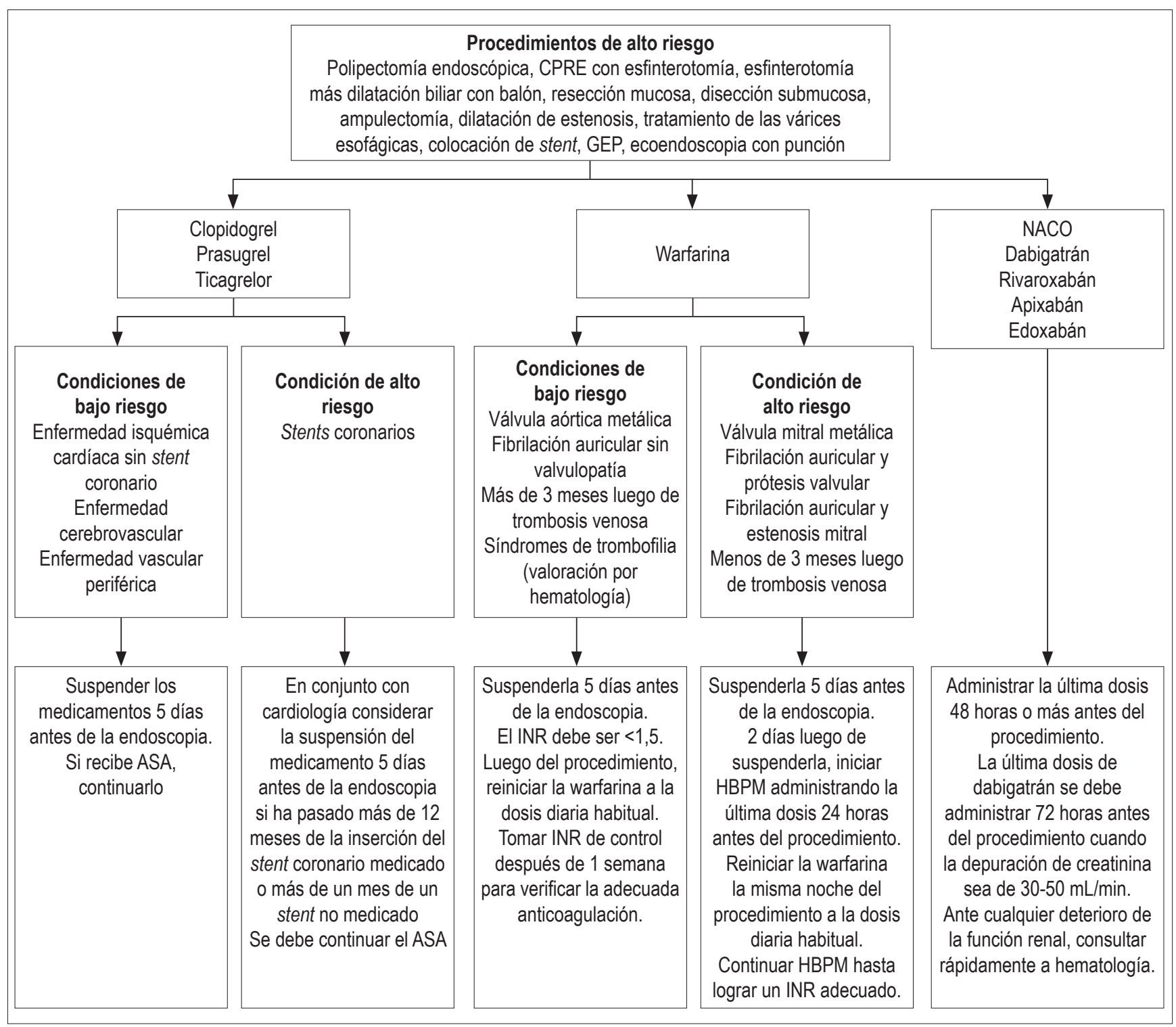

Figura 2. Uso de los medicamentos en procedimientos de alto riesgo. Modificado de: (26).

cado como factor de riesgo el uso de corriente para corte puro ya que, cuando se usa coagulación o mezcla de corrientes, el riesgo de sangrado es menor. Se considera que el uso de técnicas mecánicas, como endoloops o clips, es mejor que el uso de mezcla de adrenalina, la cual a su vez es mejor que el solo uso de adrenalina para la polipectomía. No hay estudios prospectivos respecto al uso de antiagregantes o anticoagulantes y resección de pólipos, aunque se considera que el uso de los mismos incrementa de leve a moderado el porcentaje de riesgo de sangrado, dependiendo del tipo de resección a realizar (polipectomía, EMR o ESD).

\section{CPRE}

En la práctica clínica, el riesgo de sangrado temprano o tardío cuando se realiza CPRE y esfinterotomía es del 0,1\%$2 \%$. Se han determinado como factores de riesgo asociados el sangrado observado durante el procedimiento, la coagulopatía, la iniciación de anticoagulantes durante los 3 días siguientes al procedimiento, la presencia de colangitis y la baja experiencia del endoscopista. Solo se han realizado algunos estudios en cuanto al uso de las tienopiridinas, pero no son conclusivos respecto al riesgo de sangrado. No se 
han realizado estudios en pacientes con ingesta de NACO. Algunos estudios sugieren como medida más segura la realización de dilatación de la vía biliar con balón, más que la realización de esfinterotomía.

\section{Ultrasonografía endoscópica (USE) y aspiración con aguja fina (ACAF)}

En un estudio prospectivo se determinó que no hay diferencia significativa en el sangrado que presentan los pacientes sometidos a ACAF por USE que reciben ASA en comparación con los que no. No hay estudios en cuanto a la ingesta de tienopiridinas o NACO y la realización de ACAF guiado por USE.

\section{Dilataciones endoscópicas}

Diversos estudios que evalúan el uso de dilataciones neumáticas o mecánicas no han evidenciado una importante casuística de sangrado en los pacientes sometidos a este procedimiento. No se han diseñado estudios para evaluar directamente el riesgo de sangrado asociado con dilataciones. No se han realizado estudios que evalúen las dilataciones endoscópicas en el TGI y el riesgo de sangrado en los pacientes que reciben tienopiridinas o NACO.

\section{Colocación de prótesis}

No existen estudios sobre la colocación de prótesis endoscópicas en cualquier segmento del TGI en pacientes que tomen tienopiridinas o NACO. Se considera que el riesgo de sangrado en la colocación de stents es del 0,5\%-1\%.

\section{GEP}

Algunos estudios informan que la administración de ASA no incrementa el riesgo de sangrado cuando se realiza GEP. No se han realizado estudios que analicen el riesgo de sangrado en pacientes sometidos a GEP en pacientes tratados con prasugrel, ticagrelor o NACO.

\section{Endoscopias y varices esofágicas}

En un estudio no se encontró aumento en el riesgo de sangrado al realizar EVD en los pacientes con várices esofágicas que recibían ASA. No se han realizado estudios de riesgos de sangrado en pacientes con ligadura de várices que reciben tienopiridinas o NACO, pero la recomendación es que antes de realizar el procedimiento y de ser posible se suspendan estos medicamentos.

Todos estos enunciados previos muestran las más importantes recomendaciones presentadas en la reciente publicación de las guías ESGE 2016.

\section{Coagulación y cáncer}

En la década de los 60 del siglo XIX, el médico francés Armand Trousseau describió el síndrome que actualmente lleva su nombre y se define como: Todo evento trombótico inexplicable que precede o es simultáneo al diagnóstico de un tumor maligno oculto.

La aparición de cáncer se asocia generalmente con varios síndromes trombóticos clínicos, que incluyen la trombosis venosa y arterial local y sistémica. a trombosis es a menudo la primera manifestación clínica de una neoplasia y la segunda causa de muerte en los pacientes con cáncer. La anomalía en las pruebas de coagulación in vitro se encuentra en más de $90 \%$ de los pacientes con cáncer.

Varios autores han demostrado una correlación significativa entre la incidencia de eventos tromboembólicos y un peor pronóstico de la enfermedad neoplásica, apoyando la idea de que la activación del sistema de la coagulación contribuye a la agresividad del tumor (28).

Las células tumorales activan el factor tisular (III) y la fosfatidilserina (PS), que promueve la activación de la coagulación extravascular; esto se ha correlacionado con el potencial metastásico de las células tumorales. La activación de las enzimas de la coagulación en el microambiente tumoral puede llevar a la activación de los receptores activados por proteasas (PAR), en particular PAR1 y PAR2, que se han asociado con el establecimiento de células tumorales en sitios distales al tumor primario, como se ha demostrado en melanoma, cáncer de mama y fibrosarcoma.

Dado el papel de las proteínas de la coagulación en la progresión del tumor, se ha propuesto que las mismas pueden tener un papel importante en el desarrollo de nuevas terapias antitumorales. Las estrategias terapéuticas se orientan al bloqueo de algunas moléculas efectoras de la activación de la coagulación, lo que podría atenuar el crecimiento, la angiogénesis y las metástasis tumorales (28).

\section{Importancia de la EVD y la anticoagulación}

Se ha generado un avance importante en el tratamiento de las patologías procoagulantes con nuevos y antiguos medicamentos, así como un avance diagnóstico y terapéutico en la EVD. Por tanto, es de gran importancia que el gastroenterólogo actual tenga conocimiento de este tema.

\section{REFERENCIAS}

1. Cannon CP, Rhee KE, Califf RM, et al. Current use of aspirin and antithrombotic agents in the United States among outpatients with atherothrombotic disease (from the Reduction of Atherothrombosis for Continued Health [REACH] Registry). Am J Cardiol. 2010;105(4):445-52. 
2. Ng FH, Wong SY, Lam KF, et al. Gastrointestinal bleeding in patients receiving a combination of aspirin, clopidogrel, and enoxaparin in acute coronary syndrome. Am J Gastroenterol. 2008;103(4):865-71.

3. Rodríguez A, Cáceres-Méndez E. Hemorragia de vías digestivas en el paciente anticoagulado. Rev Col Gastroenterol. $2011 ; 26(1): 39-42$.

4. Veitch AM, Baglin TP, Gershlick AH, et al. Guidelines for the management of and antiplatelet therapy in patients undergoing anticoagulant endoscopic procedures. Gut. 2008;57(8):13229. Doi: https://doi.org/10.1136/gut.2007.142497

5. ASGE Standards of Practice Committee, Anderson MA, BenMenachem T, et al. Management of antithrombotic agents for endoscopic procedures. Gastrointest Endosc. 2009;70(6):106070. Doi: https://doi.org/10.1016/j.gie.2009.09.040

6. Pérez-Gómez F. Bover R. La nueva cascada de la coagulación y su posible influencia en el difícil equilibrio entre trombosis y hemorragia. Rev Esp Cardiol. 2007;60(12):1217-9. Doi: https://doi.org/10.1157/13113924

7. Schafer AI. Coagulation cascade: an overview. En: Loscalzo J, Schafer AI (editores). Thrombosis and hemorrhage. Boston: Blackwell Scientific; 1994. p. 2-21.

8. Monroe DM, Roberts HR. Hoffman M. Platelet coagulation complex assembly in a tissue-factor initiated system. Br J Haematol. 1994;88(2):364-71. Doi: https://doi. org/10.1111/j.1365-2141.1994.tb05032.x

9. De Caterina R, Husted S, Wallentin L, et al. Anticoagulants in heart disease: current status and perspectives. Eur Heart J. 2007;28(7):880-913. Doi: https://doi.org/10.1093/eurheartj/ehl492

10. Sierra P, Gómez-Luque A, Castillo J, et al. Guía de práctica clínica sobre el manejo perioperatorio de antiagregantes plaquetarios en cirugía no cardíaca (Sociedad Española de Anestesiología y Reanimación). Rev Esp Anestesiol Reanim. 2011;58 Suppl. 1:243-50. Doi: https://doi.org/10.1016/ S0034-9356(11)70047-0

11. González M, Pérez A. Manejo de la antiagregación y anticoagulación periendoscópica: introducción a antiagregantes y anticoagulantes orales más novedosos. Rev Esp Enferm Dig. 2016;108(2):89-96.

12. Alberca de las Parras F, Marín F, Roldán Schilling V, etal. Manejo de los fármacos antitrombóticos asociado a procedimientos endoscópicos. Rev Esp Enferm Dig. 2015;107(5):289-306.

13. Soff GA. A new generation of oral direct anticoagulants. Arterioscler Thromb Vasc Biol. 2012;32(3):569-74.

14. Veitch AM, Baglin TP, Gershlick AH, et al. Guidelines for the management of and antiplatelet therapy in patients undergoing anticoagulant endoscopic procedures. Gut. 2008;57(9):1322-9.

15. Eikelboom JW, Wallentin L, Connolly SJ, et al. Risk of bleeding with 2 doses of dabigatran compared with warfarin in older and younger patients with atrial fibrillation: an analysis of the randomized evaluation of long-term anticoagulant therapy (RELY) trial. Circulation. 2011;123(21):2363-72. Doi: https:// doi.org/10.1161/CIRCULATIONAHA.110.004747
16. Kozek-Langenecker SA, Afshari A, Albaladejo P, et al. Management of severe perioperative bleeding: guidelines from the European Society of Anaesthesiology. Eur J Anaesthesiol. 2013;30(6):270-382. Doi: https://doi. org/10.1097/EJA.0b013e32835f4d5b

17. LlauJV, Ferrandis R, CastilloJ, et al. Manejo de los anticoagulantes orales de acción directa en el período perioperatorio y técnicas invasivas. Rev Esp Anestesiol Reanim. 2012;59(6):321-30. Doi: https://doi.org/10.1016/j.redar.2012.01.007

18. Aguilar N, Ramirez A, Tellez F. Manejo de antitrombóticos en pacientes que requieren procedimientos endoscópicos. Endoscopia. 2015;27(4):180-7.

19. Baron TH, Kamath PS, McBane RD. New anticoagulant and antiplatelet agents: a primer for the gastroenterologist. Clin Gastroenterol Hepatol. 2014;12(2):187-95.

20. Colantino A, Jaffer AK, Brotman DJ. Resuming anticoagulation after hemorrhage: a practical approach. Clev Clin J Med. 2015;82(4):245-56.

21. Friberg L, Rosenqvist M, Lip GY. Evaluation of risk stratification schemes for ischaemic stroke and bleeding in 182678 patients with atrial fibrillation: the Swedish Atrial Fibrillation cohort study. Eur Heart J. 2012; 33(12):150010. Doi: https://doi.org/10.1093/eurheartj/ehr488

22. Camm AJ, Kirchhof P, Lip GY, et al. Guidelines for the management of atrial fibrillation: the Task Force for the management of atrial fibrillation of the European Society of Cardiology (ESC). Eur Heart J 2010;31(19):2369-429. Doi: https://doi.org/10.1093/eurheartj/ehq278

23. Baron TH, Kamath PS, McBane RD. Management of antithrombotic therapy in patients undergoing invasive procedures. N Engl J Med. 2013;368(22):2113-24. Doi: https://doi.org/10.1056/NEJMra1206531

24. Kleiman NS. Grabbing the horns of a dilemma: the duration of dual antiplatelet therapy after stent implantation. Circulation. 2012;125(16):1967-70. Doi: https://doi. org/10.1161/CIRCULATIONAHA.112.102335

25. Kearon C, Akl EA, Comerota AJ, et al. Antithrombotic therapy for VTE disease: antithrombotic therapy and prevention of thrombosis, 9th ed: American College of Chest Physicians Evidence-Based Clinical Practice Guidelines. Chest. 2012;141(2 Suppl):e419S-94S. Doi: https://doi. org/10.1378/chest.11-2301

26. Veitch AM, Vanbiervliet G, Gershlick AH, et al. Endoscopy in patients on antiplatelet or anticoagulant therapy, including direct oral anticoagulants: British Society of gastroenterology (BSG) and European Society of Gastrointestinal Endoscopy (ESGE) guidelines. Gut. 2016;65(3): 374-89. Doi: https://doi.org/10.1136/gutjnl-2015-311110

27. Ara N, Iijima K, Maejima R, et al. Prospective analysis of risk for bleeding after endoscopic biopsy without cessation of antithrombotics in Japan. Dig Endosc. 2015;27(4):458-64. Doi: https://doi.org/10.1111/den.12407

28. Lima LG, Monteiro RQ. Activation of blood coagulation in cancer: implications for tumour progression. Biosci Rep. 2013;33(5). pii: e00064. Doi: https://doi.org/10.1042/BSR20130057 